\title{
A simple visual algorithm incorporating the components of a routine CMR study improves the determination of infarct age compared with T2-CMR alone
}

\author{
Martijn W Smulders ${ }^{1 *}$, Sebastiaan C Bekkers ${ }^{1}$, Han W Kim², Lowie M Van Assche ${ }^{2}$, Michele Parker ${ }^{2}$, \\ Raymond J Kim²
}

From 16th Annual SCMR Scientific Sessions

San Francisco, CA, USA. 31 January - 3 February 2013

\section{Background}

T2-weighted CMR is sensitive but perhaps not specific for detecting acute MI, because T2-hyperintensity can persist for months [1]. Cine and delayed-enhancement (DE)CMR may help in determining infarct age, since increased end-diastolic-wall-thickness (EDWT) and microvascular obstruction (MO) are frequently found in $<1$-month-old (acute) MI but not in 1-6-months-old (intermediate-aged) MI [1]. Given that EDWT and MO potentially resolve before T2-hyperintensity, we hypothesized that a simple, visual algorithm incorporating these components with T2-CMR could improve the determination of infarct age.

\section{Methods}

221 CMR studies were performed at various time points post-MI in 117 STEMI patients enrolled prospectively and consecutively at two centers. True MI age was known given the STEMI date. Images were scored blinded to identity and clinical information. Pre-specified markers of acute MI were: hyperintensity on T2-CMR, MO on DE-CMR, and increased-EDWT ( $>150 \%$ of remote) on cine-CMR. Our algorithm incorporating multiple CMR components was based on: 1) EDWT and MO resolve before T2-hyperintensity, 2) since T2-hyperintensity eventually disappears, T2-size becomes smaller than infarct size over time.

\section{Results}

Mean age was $58 \pm 11$ years. Table 1 shows the diagnostic performance of CMR for discriminating $<1$ from

${ }^{1}$ Cardiology, Maastricht University Medical Center, Maastricht, Netherlands Full list of author information is available at the end of the article $\geq 1$ - month-old-MI as (a) individual components, (b) basic combinations, and (c) using new algorithm. T2CMR-alone was sensitive (88\%) but not specific (66\%) for $<1$-month-old-MI resulting in only moderate accuracy $(77 \%)$. Using a later cutpoint for 'acute' MI (2-months or 3-months) did not improve accuracy since sensitivity decreased with increasing specificity. MO and increased-EDWT were very specific but not sensitive for acute MI. The basic combination of MO-or-increasedEDWT improved sensitivity (73\%) while retaining specificity (97\%). Basic algebraic combinations including T2-CMR did not improve overall accuracy since 'OR' function led to low specificity while 'AND' function led to low sensitivity. The new algorithm resulted in high sensitivity (92\%) and specificity (90\%). Accuracy (91\%) was improved compared with T2-CMR alone $(\mathrm{p}<0.001)$ and compared with basic algebraic combinations involving T2-CMR $(\mathrm{p}<0.05)$.

An additional benefit of the algorithm was the ability to identify intermediate-aged-MI (1-6-month-old). This was based on finding T2-hyperintensity-size < DE-infarct-size, and when present, patients had median infarct age of 110 days (IQR: 96, 115) (Figure 1).

\section{Conclusions}

A novel algorithm incorporating components of a routine CMR scan improves the determination of infarct age compared with T2-CMR alone. Certain CMR findings may be specific for intermediate-aged MI.

\section{Funding}

None. 
Table 1 Diagnostic performance of CMR for discriminating acute (<1-month-old) MI

\begin{tabular}{lllll}
\hline \multirow{2}{*}{ Individual CMR components } & & Sensitivity (\%) & Specificity (\%) & Accuracy (\%) \\
\cline { 2 - 5 } & T2 hyperintensity & 88 & 66 & 77 \\
\cline { 2 - 5 } & Increased-EDWT & 42 & 99 & 70 \\
\cline { 2 - 5 } & MO & 55 & 98 & 76 \\
\hline \multirow{2}{*}{ Basic combinations } & MO-or-iEDWT & 73 & 97 & 85 \\
\cline { 2 - 5 } & T2 hyperintensity or (MO-or-iEDWT) & 94 & 66 & 84 \\
\cline { 2 - 5 } & T2 hyperintensity and (MO-or-iEDWT) & 69 & 100 & 91 \\
\hline
\end{tabular}

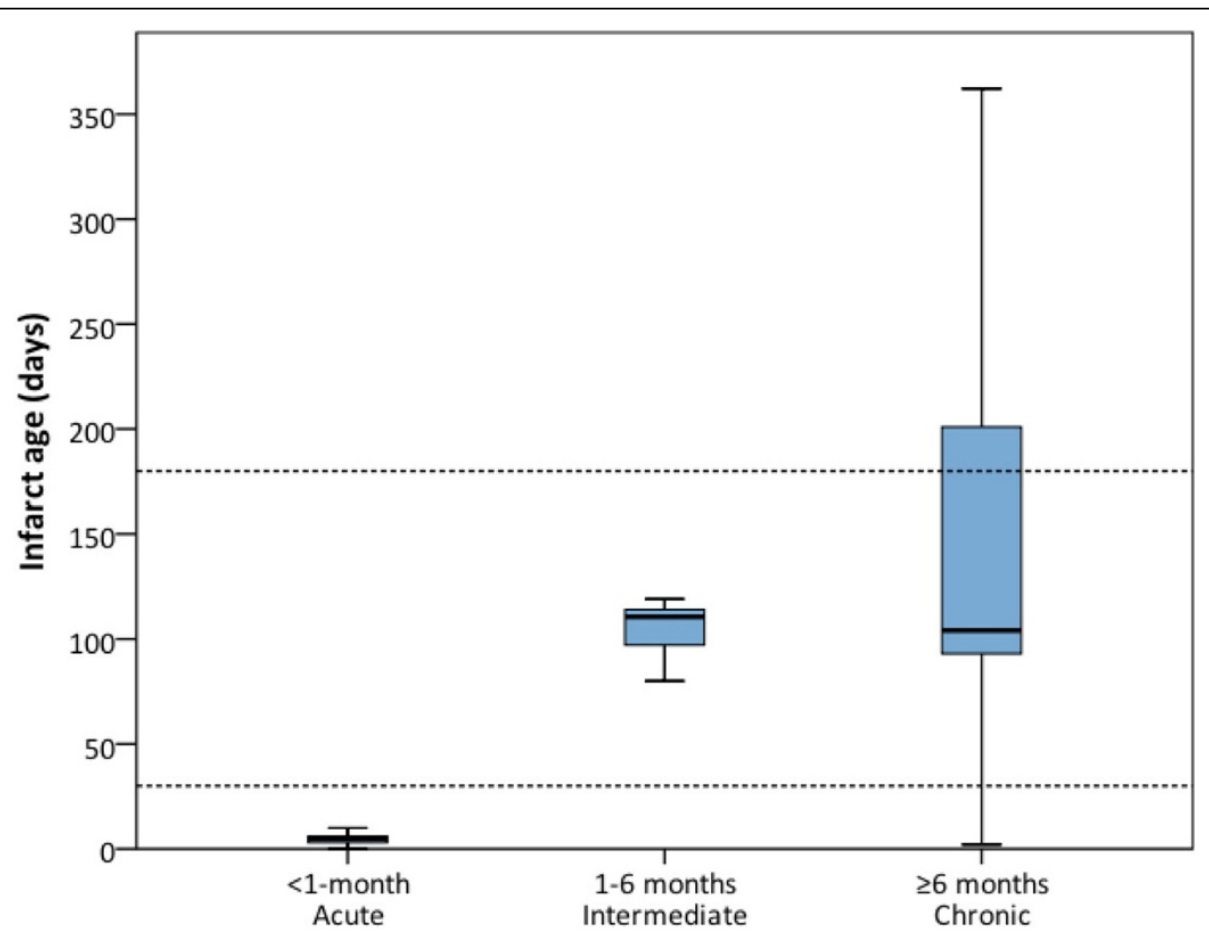

Figure 1 Range infarct age for categories based on the new algorithm

Author details

${ }^{1}$ Cardiology, Maastricht University Medical Center, Maastricht, Netherlands.

${ }^{2}$ Duke Cardiovascular Magnetic Resonance Center, Duke University Medical

Center, Durham, NC, USA.

Published: 30 January 2013

\section{Reference}

1. JCMR 2011, 13(suppl 1):068

doi:10.1186/1532-429X-15-S1-073

Cite this article as: Smulders et al: A simple visual algorithm

incorporating the components of a routine CMR study improves the

determination of infarct age compared with T2-CMR alone. Journal of

Cardiovascular Magnetic Resonance 2013 15(Suppl 1):073.

Submit your next manuscript to BioMed Central and take full advantage of:

- Convenient online submission

- Thorough peer review

- No space constraints or color figure charges

- Immediate publication on acceptance

- Inclusion in PubMed, CAS, Scopus and Google Scholar

- Research which is freely available for redistribution

Submit your manuscript at www.biomedcentral.com/submit 